\title{
LA INFLUENCIA DE LOS PASAJES EN LOS PLANES DE DESARROLLO DE SAN JUAN DE PASTO DE MEDIADOS DEL SIGLO XX
}

\author{
THE INFLUENCE OF PASSAGES IN THE \\ DEVELOPMENT PLANS OF SAN JUAN DE \\ PASTO IN THE MID-2OTH CENTURY
}

MARÍA ROSA JOJOA ZAMORA

Investigadora independiente

El texto describe cómo los pasajes que se construyeron en San Juan de Pasto a mediados del siglo $\mathrm{Xx}$ fueron significativos en su visión de ciudad moderna. En dicho escenario, los planes de desarrollo de 1945 y 1985 consagraron a los pasajes como espacios públicos y de movilidad que reactivarían el comercio, conectando la plaza fundacional con la periferia. Hacia inicios del siglo XxI, problemáticas como la densificación, la movilidad vehicular y el desempleo fueron los nuevos temas de discurso en el planteamiento urbano, relegando los pasajes a fantasías de urbanistas y arquitectos que idealizaron otro tipo de ciudad.

pasajes, modernidad, imagen, monumento
Recibido: 3 de junio del 2019

Aprobado: 5 de agosto del 2019

doi: 10.26439/limaq2020.n006.4815

This article describes how passages built in San Juan de Pasto in the mid-20th century were significant for such city to be considered a modern one. In this setting, the 1945 and 1985 development plans designated the passages as public and mobility spaces that would boost business again and connect the main plaza to the periphery. In the early 21st century, issues such as densification, vehicular movement and unemployment became the priorities of the urban approach, relegating the passages to the fantasies of urban planners and architects who idealized another type of city.

passages, modernity, image, monument 


\section{LOS PASAJES EN CONTEXTO: LA CIUDAD MODERNA EUROPEA} Y LATINOAMERICANA

Existen espacios como los pasajes que sin estar entre los más sobresalientes y estudiados en la ciudad son importantes para entender otro tipo de dinámicas de movilidad. Desde la propia etimología del término "pasaje", que según señala el Diccionario de la lengua española proviene del verbo latino passare, que significa "dar pasos" (Real Academia Española [RAE], 2020); y del sufijo francés aje que equivaldría a "acción de", se comprende la función que estos cumplen en la ciudad. Para contextualizarlos en el lenguaje de la urbe, los pasajes siempre darán paso de una calle a otra generando circulaciones distintas. En el escenario urbano los pasajes se integran a la traza, sin haber sido pensados como parte de ella, al romper la rigidez de la manzana cortándola vertical u horizontalmente; de este modo permiten la conexión entre calles, funcionan espacialmente como lugares de transición entre lo público y lo privado, y crean una permeabilidad peatonal hacia su interior. El uso al que se destine depende de su ubicación y diseño. Como conjunto, los pasajes poseen la fuerza para proyectar una nueva traza de circulación para los peatones que va en contravía respecto de la estructura urbana establecida, lo cual genera toda una experiencia citadina alrededor de ellos.

Su aparición en la escena urbana se remonta a la industrialización europea, en el París de la segunda mitad del siglo xIx; un París que atravesaba un rápido crecimiento urbano debido a políticas de estado y al exponencial desarrollo comercial. En ese contexto, Napoleón III proyectaría la construcción de una ciudad moderna con el ferviente deseo de convertirla en la primera ciudad industrial. Al respecto, Sigfried Giedion (1982) en su libro Espacio, tiempo y arquitectura menciona: "La metrópolis de la era industrial adquirió súbitamente su forma característica en París entre 1850 y 1870. En ninguna otra ciudad de ese período avanzaron con tal ímpetu los cambios producidos como consecuencia del desarrollo industrial" (p. 706).

En este escenario, los primeros pasajes aparecen como un reflejo del buen desarrollo comercial por el que atravesaba París; estos se convirtieron en una respuesta económica y social, a la vez que en espacios que conectaron áreas públicas y comerciales de la ciudad de inicios del siglo xIx y se expandieron por Alemania, Inglaterra, España, entre otros países. Su concepto principal surgió de traspasar la manzana de un lado a otro, convirtiéndose en ejes de acción para generar una espacialidad distinta, de lujo, confort y comercio en su entorno urbano. A nivel arquitectónico sugerían un intento de monumentalización, fruto de la ciudad moderna europea.

Antes de hablar de la incidencia de los pasajes en algunas ciudades del territorio latinoamericano, conviene hacer una breve introducción al acontecer urbano de esa época. Andreas Hoffer (2003), en su libro Karl Brunner y el urbanismo europeo en América Latina, expresa que Latinoamérica, durante la primera mitad del siglo xx, estuvo dedicada a la consolidación de nación, al desarrollo de la economía y al nacimiento de la industria. Esas transformaciones hicieron 
que las ciudades evidenciaran la necesidad de planificar la expansión urbana. En ese escenario, la visión de una ciudad moderna estaría influenciada por la necesidad del desarrollo, al igual que por abandonar su imagen de ciudad colonial. El mismo autor señala que la ciudad moderna latinoamericana encontró en los modelos urbanos europeos la visión de ciudad a futuro, los cuales estuvieron ligados a principios estéticos, higienistas y de circulación de finales del siglo XIX.

Para Arturo Almandoz (2007) la planificación y proyección de ese nuevo modelo de ciudad se manifestaron en países como Argentina, Chile, Venezuela y Colombia, los cuales contaron con la asesoría de arquitectos y urbanistas extranjeros, quienes trajeron nuevas teorías y un modelo de ciudad europea y norteamericana al contexto latinoamericano. Este fue el caso de Karl Brunner, quien visitó Santiago de Chile y Bogotá, o Le Corbusier quien hizo un recorrido extenso por gran parte de Latinoamérica llevando sus principios arquitectónicos y un modelo de ciudad moderna europea para la planificación de la ciudad del futuro.

En dicho intento de construir una urbe del porvenir aparecen los pasajes con una connotación distinta a la europea; en Latinoamérica fueron construcciones con fines comerciales que permitían el libre tránsito de personas, lo cual era una opción para acortar caminos. Sin embargo, no llegaron a representar el esplendor de la ciudad moderna europea de finales del siglo xIx. En países como Argentina y Chile el valor agregado de los pasajes radicó en su inclusión en los planes de desarrollo como parte fundamental para el crecimiento y la movilidad del centro histórico, en Bogotá y Cartagena fueron construcciones aisladas dedicadas a incentivar el comercio de la época.

En el caso de San Juan de Pasto, los primeros pasajes surgieron como parte de una ideología de inicios del siglo xx que priorizaba la construcción de monumentos arquitectónicos. Posteriormente, en la década de los cuarenta del siglo pasado, estos se convertirían en espacios urbanos significativos para la planeación urbana. Culminada la década de los setenta, aparecerían los pasajes comerciales obedeciendo al carácter comercial de la región de Nariño (ver figura 1).

\section{LOS PASAJES EN SAN JUAN DE PASTO}

Si bien los pasajes en Europa fueron fieles representantes de la ciudad moderna de finales del siglo XIx, en el caso de San Juan de Pasto su concepto estará relacionado con la actividad que se desarrolla en el entorno urbano. La arquitecta María Beatriz Aguirre (2009) en su tesis doctoral Los pasajes y galerías comerciales de Santiago de Chile, como tránsito urbano hacia la modernidad, relaciona el concepto de pasaje con la función de los edificios existentes y con las articulaciones espaciales que se generen: 


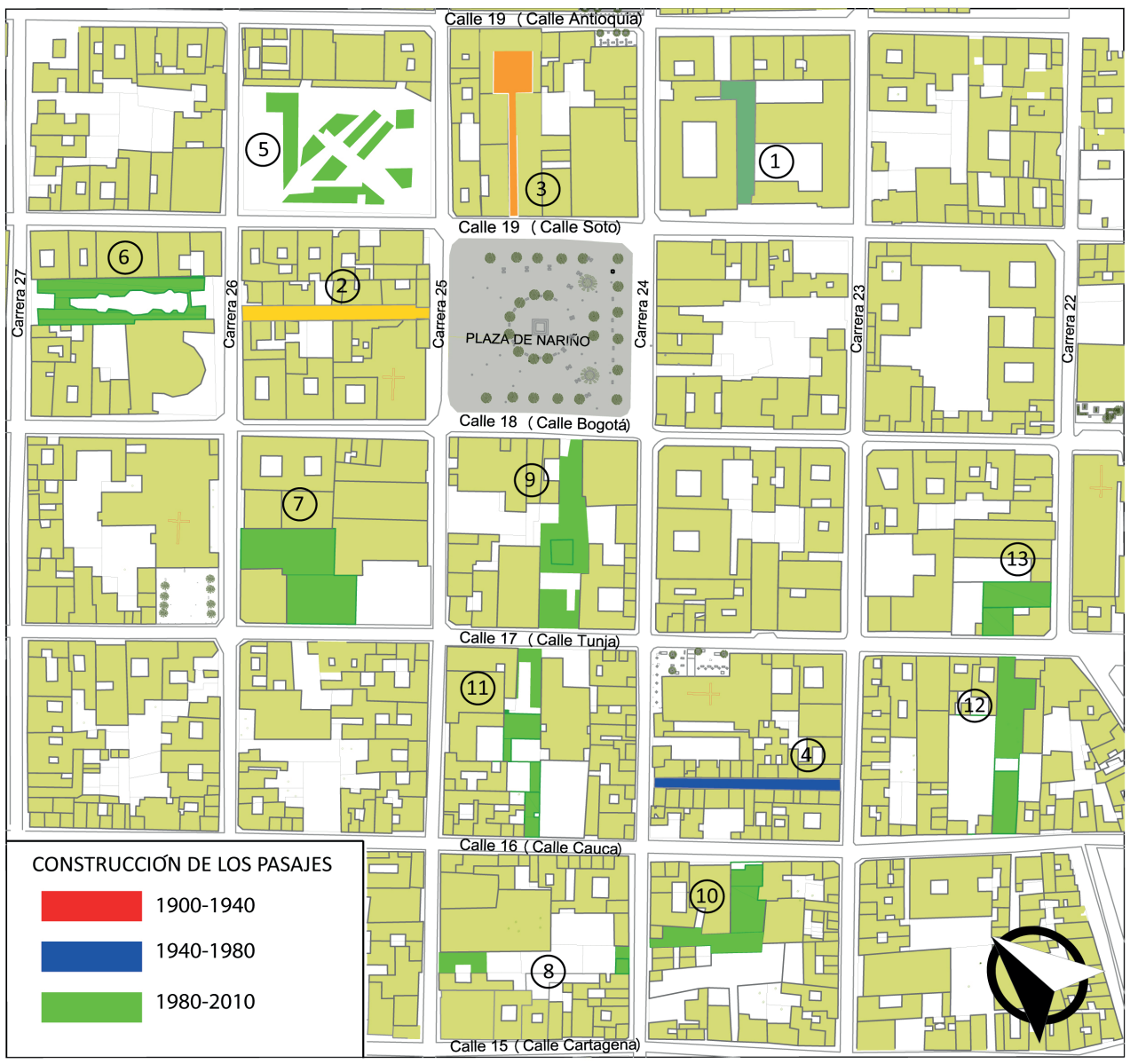

1) Paso de la Gobernación de Nariño

2) Pasaje Corazón de Jesus

3) Pasaje de la Carrera 24

4) Pasaje del Dorado

5) Centro Comercial Sebastián de Belalcazar

6) Centro Comercial Galerias
7) Pasaje el Liceo

8) Pasaje el Zaguan del Lago con Centro Comercial Astrocentro 24

9) Centro Comercial la 17 con Centro Comercial los Andes

10) Centro Comercial San Agustín con paseo de Dorado

11) Pasaje la 17 con Ventas populares la 16

12) Pasaje Torres la 17

13) Pasaje Orient
Figura 1. Red

de pasajes

existente en los

alrededores del

centro funda-

cional (plano de

llenos y vacios

de Pasto, 2014)

Elaboración propia
Esta tipología se fue haciendo cada vez más frecuente con la importancia y centralidad que adquirieron ciertas funciones en la ciudad ... Por ejemplo, la representación de las funciones cívicas, financieras e institucional que toman preeminencia y exigiendo el diseño de otros escenarios capaces de responder adecuadamente a la complejización de las estructuras edilicias existentes. (p. 191)

Es necesario destacar que si bien un pasaje, espacialmente, podría ser el mismo en parámetros formales, las significaciones cambian de un lugar a otro. Como se estudió anteriormente, en las principales ciudades un pasaje puede estar 
asociado a las grandes construcciones, al esplendor y evolución de la ciudad moderna, en otras podría ser el producto de un sistema económico, de esas interacciones sociales y económicas de compra y venta que se traducen en espacios. Sin embargo, si algo tienen en común es que su epicentro va a estar ligado a la manzana y a las transformaciones que eventualmente van a surgir en ella.

En San Juan de Pasto se encuentran dos tipos de pasajes como se conocen en el presente, los primeros encuentran sus inicios en las fracturas de manzana y dan realce al monumento arquitectónico, como lo son el pasaje de la Gobernación de Nariño, el Corazón de Jesús y el de la Carrera 24. En segunda instancia se encuentran los pasajes ubicados en el centro histórico y que surgieron a finales del siglo xx como fruto del crecimiento económico de la ciudad.

\section{EL PASAJE DE LA GOBERNACIÓN DE NARIÑO}

La Gobernación de Nariño se localizó en la esquina o cruce de la antigua calle Buenaventura (hoy Carrera 23) y la calle 19 o calle Soto. Se concibió como la primera de una serie de construcciones hechas para dotar a la ciudad de un ornato público digno de la época. Asimismo, esta edificación se enrumbaría por la grandeza del estilo republicano, utilizando la tecnología del ladrillo como elemento primordial para sustituir la tapia.

Hasta el siglo XIx las edificaciones más sobresalientes del casco urbano que venían desde la colonia fueron las iglesias y los conventos. En esa época, San Juan de Pasto no contaba con un edificio institucional de relevancia y es en 1910 que, por impulso del gobernador Gustavo Guerrero Herrera, la construcción del primer edificio de carácter civil se hizo realidad. La obra arquitectónica debía imprimir relevancia en el panorama arquitectónico de la ciudad; de allí nació el empeño por convertir la estructura del nuevo edificio en algo singular utilizando en su diseño una tipología y morfología atípicas en el casco urbano de la ciudad (ver figura 2).

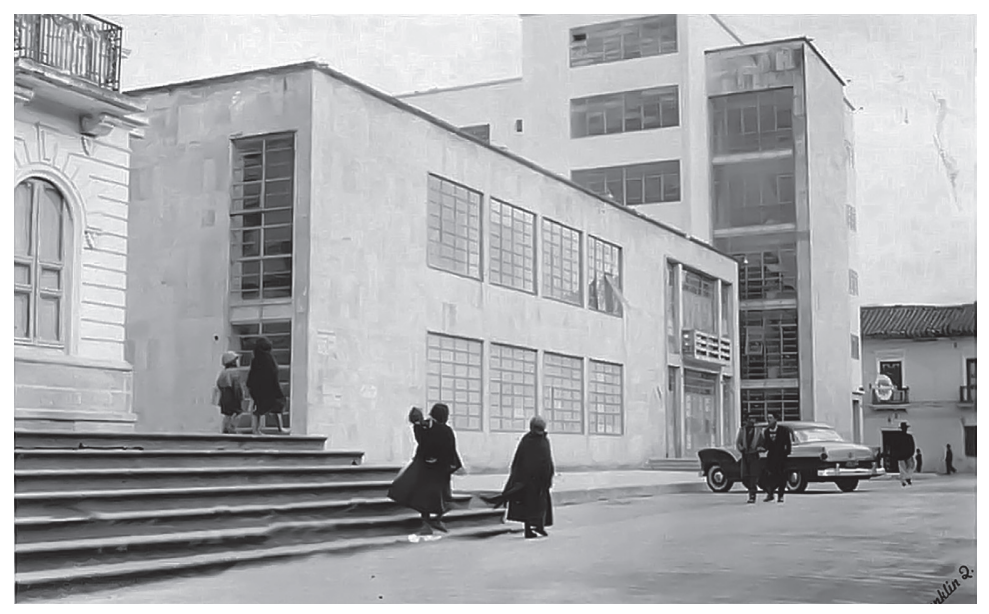

Figura 2.

Fachada principal del

Palacio de Justicia donde se aprecia la puerta de acceso del pasaje de la Gobernación de Nariño (plaza fundacional, 1950-1960)

Fuente: Franklin. Q. Archivo fotográfico del Centro Cultural del Banco de la República, Pasto, San Juan de Pasto, Nariño 
En la concepción formal del edificio cobra valor un paso de uso peatonal restringido que rodea la edificación con el propósito de jerarquizar la construcción. Si bien este tipo de espacio se contempló para uso exclusivo de la Gobernación de Nariño, con el pasar del tiempo se convirtió en un foco de propuestas que lo visualizaron como un posible pasaje que se conectara con la plaza central.

En 1941 Karl Brunner contempló su uso dentro del plan denominado "Pasto Futuro" como parte de la estrategia de movilidad, pasando su función de uso privatizado a uso abierto y denominándolo pasaje. Posteriormente, en 1944, el arquitecto Bruno Violi diseñó el Palacio de Justicia respetando su continuidad; así, lo que antes era una L se convirtió en una T que unió a los dos edificios. Para el arquitecto Alejandro Paz, antiguo director de obras de la Gobernación de Nariño, el objetivo era claro: "El palacio judicial planeó que la T fuera abierta, pero eso nunca pudo hacerse una realidad" (A. Paz, comunicación personal, 4 de octubre del 2013). Los planes reguladores de 1968 y de 1985 consideraron mantener su uso como espacio abierto manteniendo viva la imagen de pasaje peatonal. Sin embargo, con el transcurrir del tiempo, el uso privatizado del edificio de la gobernación enterró ese ideal de pasaje.

\section{PASAJE CORAZÓN DE JESÚS}

El pasaje Corazón de Jesús se localiza entre la calle Bogotá (hoy calle 18), la calle Soto o calle 19 y frente a la calle Santander (actual carrera 24). Fue inaugurado entre 1928 y 1934, convirtiéndose desde esa época en una de las construcciones más emblemáticas de la ciudad, no tanto por su uso institucional, sino por su monumentalidad arquitectónica que sobresale en el conjunto urbano del centro histórico. El proyecto se destaca por tener un arco abovedado en doble altura (inspirado en el Arco del Triunfo romano) que se enfrenta a la plaza principal. Como menciona Urresty (2000) en la Historia urbana de Pasto la construcción no es del todo adecuada con su entorno, porque "al tratar de adaptar el modelo extranjero en un lote angosto dio como resultado una inadecuada disposición interior con habitaciones estrechas e incómodas" (p. 235). Se podría inferir que este pasaje nace como una construcción decorativa que carece de funcionalidad práctica porque su función era más de orden simbólico.

Como elemento urbano, este paso público se extiende hacia la plaza mediante un arco romano abovedado en doble altura; su composición parte de una fractura de manzana que permite crear una entrada y salida hacia la plaza principal. Asimismo, la resolución arquitectónica del pasaje Corazón de Jesús, que sería el segundo que tendría la ciudad, tiene una clara intención de proyectar al monumento con vistas al centro histórico.

Este lugar de tránsito se construyó como sede del Palacio Episcopal, sin embargo, debido a las demandas funcionales no pudo ser ocupado por sus limitaciones de espacio y se destinó a alquiler para diversos usos. El primer 
cambio de uso ocurrió en 1934 cuando dejó de ser sede episcopal y pasó a ser oficina de correos y telégrafos; posteriormente, se destinó para comercio mixto (ver figura 3 ).

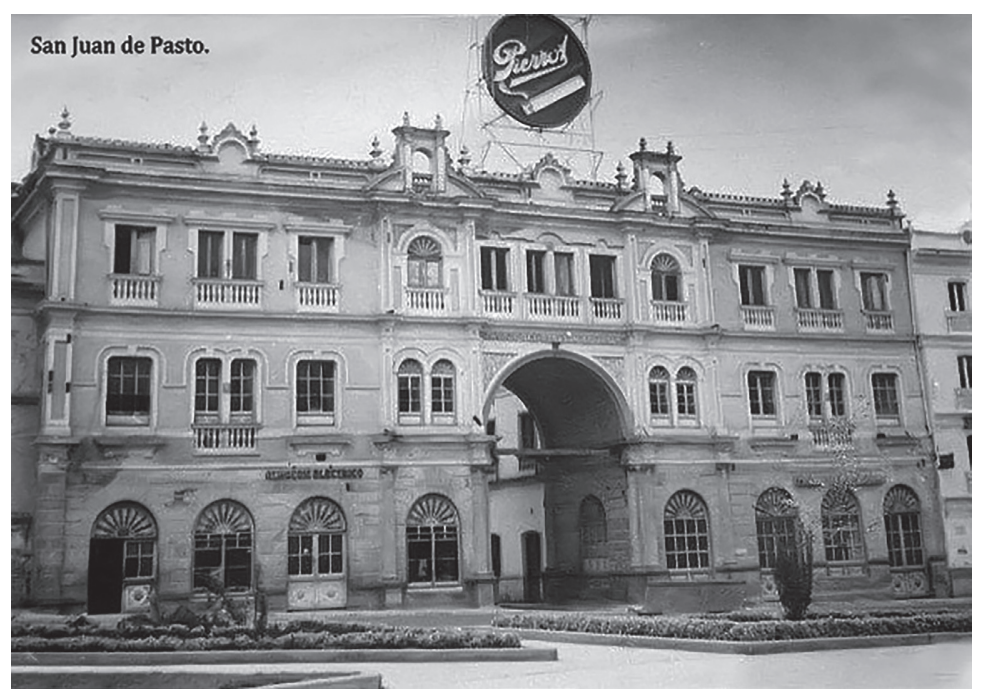

Figura 3.

Fachada

principal del

pasaje Corazón

de Jesús. Se

aprecia la aper-

tura del pasaje

como espacio

público (plaza

fundacional,

1940-1950).

Fuente: Archivo

fotográfico del

Centro Cultural

del Banco de la

República, Pas-

to, San Juan de

Pasto, Nariño

El pasaje Corazón de Jesús cobra vida y significado cuando se abre para uso peatonal. Un sueño que, como se verá más adelante, se contempló por largo tiempo en la mayoría de los planes de desarrollo. Karl Brunner lo visualizó como un espacio que comunicaría el centro con el resto de la ciudad; el plan de 1968 lo contempló como parte del proyecto de movilidad del centro histórico, hasta que el modelo del año 1985 lo catalogó no solo dentro del patrimonio a conservar, sino como parte de la red de circulación peatonal que rehabilitaría el centro. En la actualidad, el pasaje Corazón de Jesús es el único en la ciudad que logra materializar ese ideal de pasaje, manteniendo su carácter público con uso comercial de bajo impacto y circulaciones que vinculan a la plaza con las calles circundantes.

\section{PASAJE DE LA CARRERA 24}

A fines de los años treinta del siglo xx se inició la construcción de este pasaje junto al Banco de Colombia en la antigua Casa Consistorial, ubicada aproximadamente en el solar central de la manzana oriental del marco de la plaza central de la ciudad (actual plaza de Nariño), frente a la antigua calle Soto (actualmente la calle 19) entre la antigua calle Azuero (hoy carrera 25) y la calle Santander (hoy carrera 24).

El diseño del Banco de Colombia se rigió por el estilo moderno de la época planteando una construcción que armonizara con la imagen del centro 
histórico y la del resto de edificios en conjunto. Contó, además, con un pasaje lateral que separaba al edificio de su colindante y atravesaba la manzana totalmente. Sin duda, este hecho fue un fenómeno atípico que cambiaría considerablemente la morfología del centro histórico de San Juan de Pasto.

Registramos complacidos la construcción del pasaje del Banco de Colombia. Hemos leído en la prensa algunas críticas referentes a este edificio cuya construcción marcha con rapidez. A nuestro modo de apreciar las cosas creemos que la belleza estará en conjunto cuando la obra quede completamente terminada (Ilustración Nariñense, 1941, p. 13).

Este espacio, que se convirtió en el primer intento real de construcción de pasaje como un gesto de ciudad moderna, sería la primera aproximación a una fractura de manzana como generadora de cambios en la espacialidad del centro histórico. El pasaje que se intentó construir, lejos de monumentalizar el edificio, se convertiría en una puerta urbana inacabada que generaría una circulación que conectaría la plaza con el colegio San Francisco Javier, estableciendo una conexión entre el centro y las calles circundantes (calle 20 y calle 21). Su función desde sus inicios fue prevista como un pasaje que cruzaría de un lado al otro, siendo uno de los primeros elementos que reflejaría los sueños del "Pasto Futuro" (ver figura 4).

Figura 4

Fachada prin-

cipal del Banco

de Colombia

donde se aprecia

la apertura del

pasaje de la

Carrera 24 (Pla-

za fundacional

1940-1945)

Fuente: Archivo

fotográfico

Centro Cultural

del Banco de la

República, Pasto,

San Juan de

Pasto, Nariño

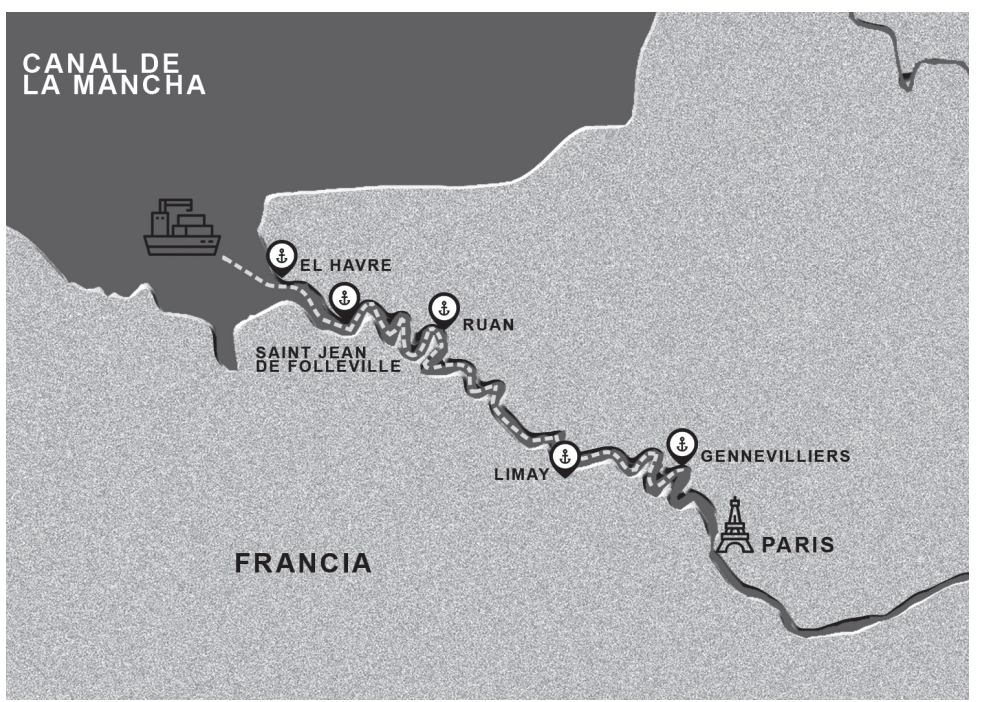

Esta intervención sería protagonista de la "Ciudad Futuro", propuesta por Karl Brunner en 1941, en la cual el pasaje se convertiría en pieza clave de la transformación del centro histórico. Los posteriores planes de desarrollo (prestar particular atención al POD 85) lo utilizarían como una solución de peatonalización de la plaza fundacional que la uniría con las vías colindantes. 


\section{LOS PLANES DE DESARROLLO, UNA VISIÓN A FUTURO PARA SAN JUAN DE PASTO}

Para la década del treinta del siglo $\mathrm{xx}$, los tres pasajes cobrarían importancia en un escenario con problemáticas sociales y urbanas que impedían a la ciudad avanzar a la modernidad. La ciudad de esa época padecía de calles sin pavimentar, falta de infraestructura y un alcantarillado en mal estado. Es esa atmósfera la que lleva a la ciudad a la búsqueda de un cambio que se percibía en los ideales urbanos de producción constructiva, de embellecer la ciudad, de higienización, de confort y salubridad.

La llegada del IV centenario fue la plataforma para la construcción de esos ideales; de este modo, se inició un plan de transformación que vinculaba progreso, higienización, embellecimiento y movilidad dejando atrás los trabajos fragmentados como pavimentación de calles y alcantarillado. Fue ese el momento de visualizar la ciudad como parte de un todo, como un acontecimiento que los ciudadanos empezarían a pensar en colectivo. "Si Pasto puede ofrecer en el día clásico de la celebración de su iv centenario la terminación de sus obras centenarias, habrá logrado el mejor tiempo y habrá satisfecho un anhelo largamente sentido" (periódico La Radio, 1935, p. 3). Este anhelo quedó plasmado en el plan regulador propuesto por la Sociedad de Mejoras Públicas (SMP). El cual, con el tiempo, se convertiría en el plan "Pasto Futuro" de 1941. En él se darían las pautas para llevar a la ciudad por un camino ordenado, de vías modernas, edificios y proyecciones que cambiaran radicalmente la imagen de la ciudad.

\section{EL PLAN BRUNNER 1941, LOS PASAJES ENTRAN EN ESCENA}

Karl Brunner llegó a Colombia contratado por la alcaldía de Bogotá, en diciembre de 1933, como primer director del Departamento Municipal de Urbanismo. Asumiendo este cargo se involucró en grandes proyectos de reforma, ensanche y mejoramiento de infraestructura, según lo menciona Hoffer (2003). Las propuestas urbanísticas que realizó en Colombia empezaron en 1935 cuando trabajó como asesor del gobierno para generar distintos planes de desarrollo en la mayoría de las ciudades del país. Se destacaron los planes para Barranquilla, Medellín, Manizales, Buenaventura, Cali, Santa Martha y San Juan de Pasto.

En el informe presentado a Rafael Erazo Navarrete en 1941, Karl Brunner presentó la propuesta "Ciudad Futuro" para San Juan de Pasto; en ella se explica detalladamente las mejoras y reformas urbanas y arquitectónicas que deben realizarse en la ciudad, especialmente la transformación y desarrollo de la zona de expansión y de la zona centro. Para Brunner fue de vital importancia introducir vías radiales que se comuniquen con la retícula fundacional, esto como medio para facilitar el ensanche urbano en distintas calles. También amplió las arterias principales para dar paso al vehículo, sobre todo entre las calles 18 y 19 donde la afluencia de gente hizo necesaria una holgura entre ellas. 
Uno de los aspectos más interesantes de esta propuesta fue la apertura de pasajes con diversidad de usos en los alrededores de la plaza fundacional de la ciudad. Karl Brunner (1976) menciona:

En la parte central de la ciudad se adelantan construcciones que consultan la apertura de pasajes a través de las manzanas; así, por ejemplo, en el predio del Banco de Colombia con dirección al Colegio Javeriano y en la manzana del Palacio Municipal donde se proyecta el hotel. Estas aperturas ayudarán mucho al fácil desarrollo de la circulación de transeúntes y aumentarán frentes disponibles para el comercio, .... Un poco más difícil resulta una apertura de vía a través de manzanas edificadas, cuando su dirección no es paralela a las calles o Carreras; sin embargo, ciertas circunstancias pueden aconsejar que se afronten las posibles dificultades. (p. 377)

prototipo de la

propuesta)

Fuente:

revista Ilustración

Nariñense, 1938 ,

San Juan de

Pasto, Nariño

Desde el punto de vista conceptual, el significado de "pasaje" se inserta como un elemento articulador urbano. El plan los proyecta como espacios de circulación peatonal que ayudarían a conectar la plaza con la mayoría de sus frentes, serían fuentes de comercio e implementarían una nueva imagen al centro histórico (ver figura 5).

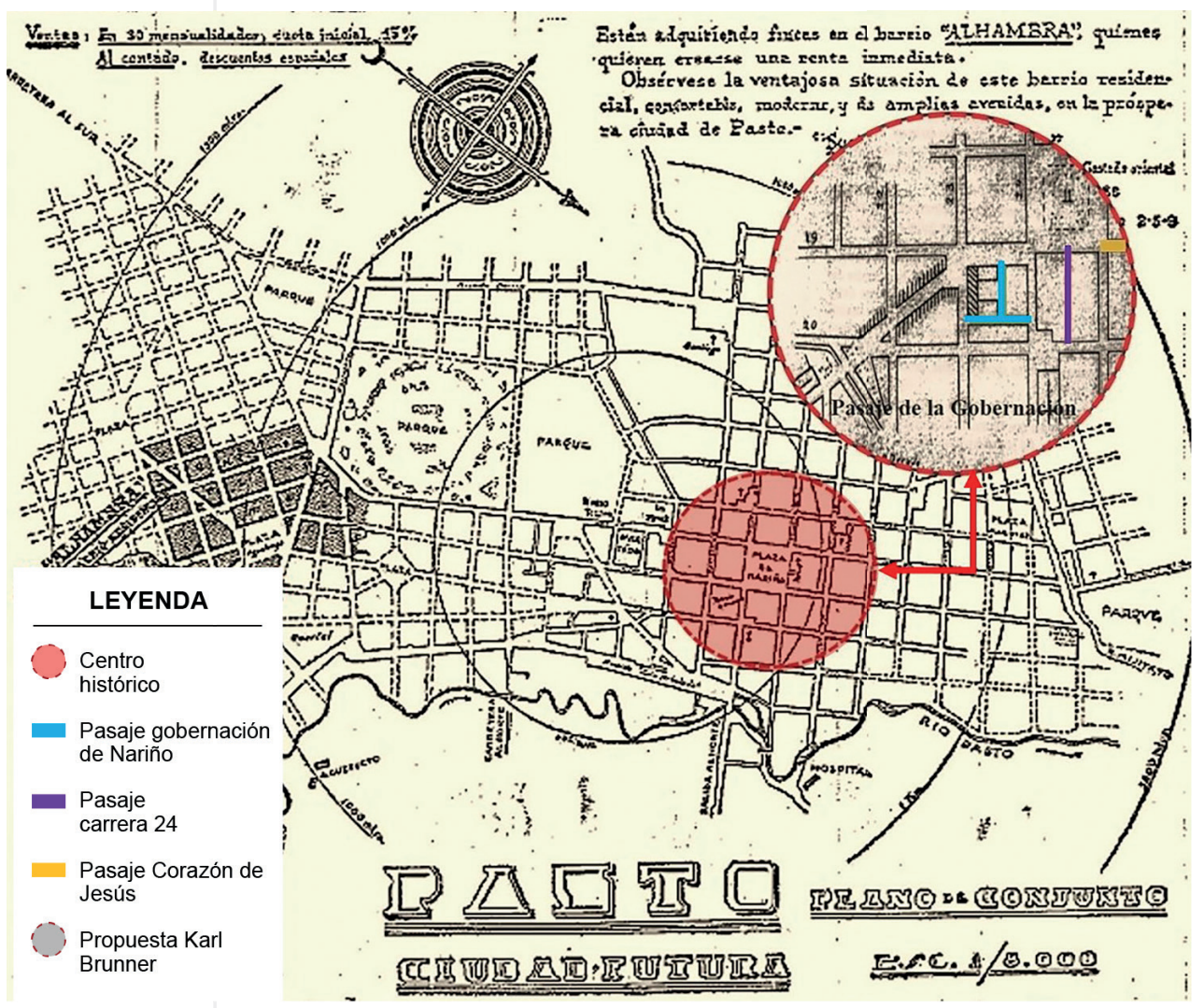


La visión de aperturas de manzanas y pasajes como parte de la transformación del centro histórico propuesto por Brunner fue el detonante para que estos espacios empezaran a ser recurrentes en los planes de desarrollo; de este modo, perdieron su connotación de espacios residuales, producto de las construcciones más emblemáticas del centro histórico, para pasar a ser parte de la estructura de la traza existente. Los pasajes, a través de la planificación urbana, comenzaron a tener vida en los sueños de la ciudad convirtiéndose en soluciones a futuro para la circulación peatonal del centro histórico y en una respuesta a una ruta que pretendía integrar estos espacios con vías alternas en la ciudad.

Lastimosamente este plan no se ejecutó, burocracias lastraron su camino hasta archivarlo. Si el tema de los pasajes estaba en el mapa durante el trascurso de los años cuarenta y cincuenta, estos se fueron extinguiendo al priorizar el crecimiento urbano de la ciudad. Embellecer el centro histórico dejó de ser importante y se dio paso a asuntos de primera necesidad como vivienda y servicios. Sin duda otros serían los rumbos que tomaría el municipio, dejando a los pasajes como un sueño de la "Ciudad Futuro" de Brunner.

\section{EL PLAN DE LA UNIVERSIDAD NACIONAL: 1986}

El plan de desarrollo propuesto por la Universidad Nacional en 1985 contó con la dirección principal del arquitecto Manuel García Camacho, profesor de urbanismo especializado en el área de planificación urbana de dicha universidad.

La propuesta de intervención en el centro histórico se organizó a través de la recuperación de la función de la plaza principal como un lugar de "congregación” de la comunidad. Así, el planteamiento urbano de Manuel García propuso un paseo llamado San Juan de Pasto, con ejes peatonales que articularían los diferentes sitios de interés patrimonial del centro fundacional. Este paseo rescataría la idea de Karl Brunner y su plan "Ciudad Futuro", abriendo los pasajes ubicados en la Gobernación y en el Banco de Colombia; además, el plan generó una propuesta con base en ellos.

Sobre los pasajes peatonales dice:

La posibilidad de establecer pasajes que atraviesen las manzanas en cualquier dirección no debe implicar la densificación exagerada de las partes de manzana resultantes. La unidad básica de intervención debe ser la manzana completa, construida en su periferia y con un vacío en el centro, que debe mantenerse descubierto y formar parte del pasaje para integrarse a la trama de espacios públicos. En cada caso particular deben establecerse los límites para el nuevo frente que adquieran los predios localizados sobre el pasaje y sobre el centro de la manzana.

Los aislamientos posteriores existentes se convertirían en el nuevo espacio público, evitando la excesiva ocupación resultante en casos como el pasaje el dorado. 
Figura 6 .

Plano sección

tipo pasajes

peatonales de

1986 diseñado

por la Universi-

dad Nacional;

en él se observa

la propuesta de

diseño para los

pasajes ubicados

en el centro

histórico de la

ciudad de Pasto.

Fuente: Roberto

Erazo Nava-

rrete, Plan de

Ordenamiento de

Desarrollo 1986.

San Juan de

Pasto, Nariño.
De cualquier forma, este tipo de intervención implica un aumento en la posibilidad de ocupación de los predios que componen la manzana y de su rentabilidad al tener doble frente sobre el espacio público, lo que de por sí es ya un aliciente para el inversionista y para los propietarios de los demás predios de la manzana. En general, deben aplicarse, además, los criterios ya definidos para las plazuelas en cuanto a amueblamiento, pisos, iluminación, etcétera (ver figuras 6 y 7). (Universidad Nacional de Colombia, 1985, p. 69)

PASTO - ÁREA CENTRAL

\section{SECCIÓN TIPO PASAJES PEATONALES. 1986}
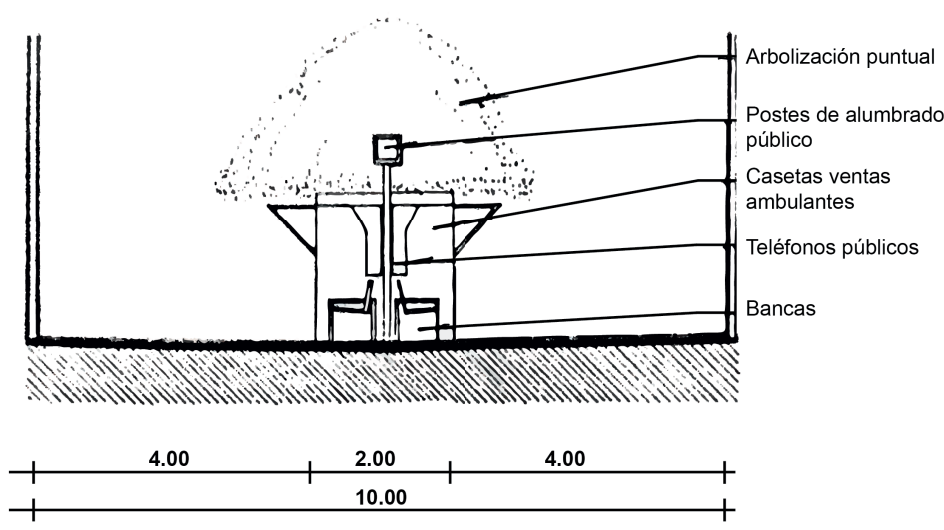

SECCIÓN TRANSVERSAL 8

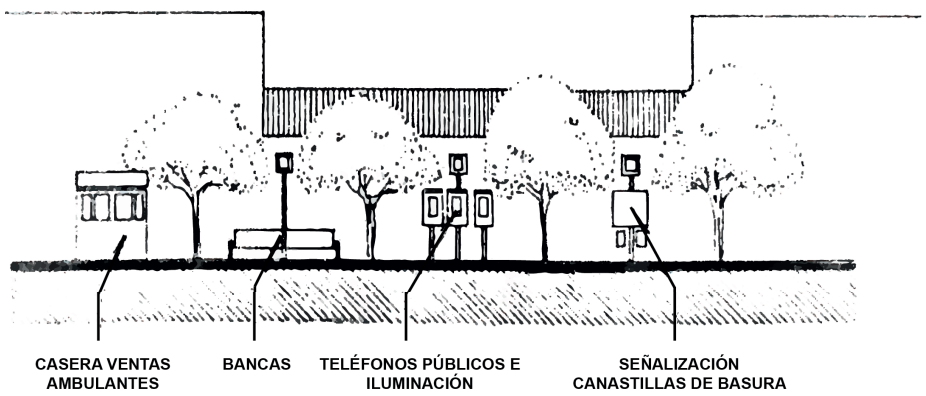

Si bien la peatonalización del centro histórico se realizó de la mano con nuevas estrategias de transporte, apertura de vías y nuevas conformaciones de zonas verdes, la inclusión de pasajes al plan de movilidad abría la posibilidad de generar espacios públicos en los centros de manzana; además, los pasajes se convertirían en espacios que podrían fortalecer los predios aledaños en cuanto a rentabilidad y usos. Se podría concluir que el plan de ordenamiento de 1985 

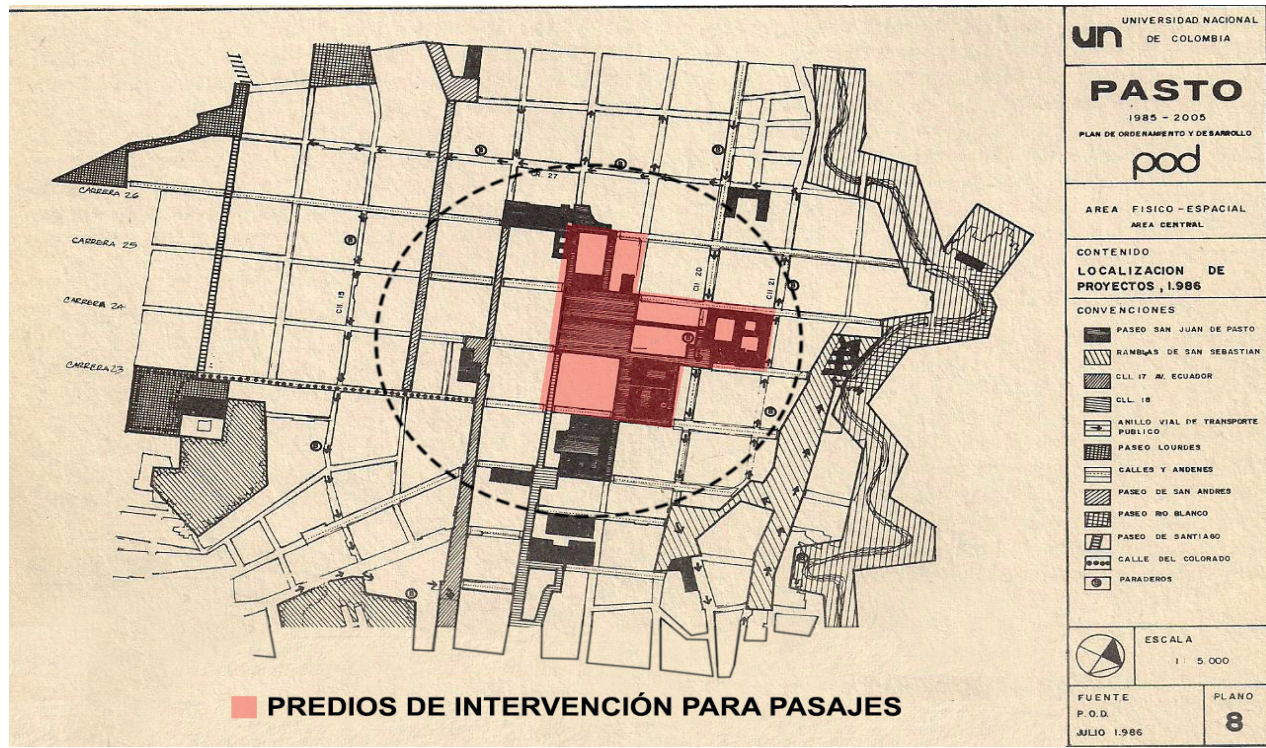

rescató el potencial de los pasajes como espacio público, contextualizándolos con los retos de la ciudad del siglo xx. Lastimosamente, el paseo San Juan de Pasto no logró hacerse realidad dejando nuevamente a los pasajes como un sueño de la ciudad moderna.

\section{CONCLUSIONES}

La construcción de la ciudad moderna de San Juan de Pasto estuvo ligada a la evolución de los pasajes. Desde que se empieza a hablar de modernidad, la imagen de la ciudad daría un giro en la concepción del edificio, la plaza y sus calles. En ese contexto aparecen los primeros pasajes, piezas clave para entender que San Juan de Pasto vivió dos momentos de transformación: el primero "la ciudad de los monumentos" (1900-1930) donde se evidenció la búsqueda del embellecimiento de la ciudad en lo arquitectónico y urbano a inicios del siglo xx; en ese escenario, los pasajes nacen como espacios que jerarquizarían las construcciones de valor simbólico y patrimonial en el centro histórico. El segundo momento está ligado a la "ciudad planeada" (1930-1985), esa que promovió unos ideales urbanos y arquitectónicos para la planeación y construcción de una ciudad. En ese periodo, los pasajes se proyectaron como elementos sobrepuestos a la traza del centro histórico, que introducen nuevas posibilidades de uso y circulación en el sueño que fue el "Pasto Futuro".

Los tres pasajes de estudio (el de la Gobernación de Nariño, el Corazón de Jesús y el de la Carrera 24) pasaron por un largo proceso de transformación hasta adquirir la connotación espacial de pasajes a través de los planes de desarrollo y a causa de los ciudadanos. A inicios del siglo xx, cuando se construyeron

Figura 7

Plano "Ciudad

Futuro" de 1986

diseñado por

la Universidad

I Nacional; en él

se observa el

segmento de la

propuesta para

centro funda-

cional con la

trama de pasajes

(pasaje de la

gobernación de

Nariño, pasaje

corazón de Jesús

y pasaje de la

Carrera 24).

Fuente: Roberto

Erazo Navarrete,

1986, San Juan

de Pasto, Nariño 
los dos primeros, se consolidaron como fracturas y pasos, puesto que no traspasaban la manzana cerrada en su totalidad o eran espacios privatizados. En la década del cuarenta, con la construcción del pasaje de la Carrera 24 y con la visión del urbanista y arquitecto Karl Brunner, los tres pasajes aparecieron en el mapa como posibles vías de circulación peatonal. Fueron, entonces, los planes de desarrollo quienes les dieron la connotación de pasajes; y con esta condición adquirida (a excepción del pasaje Corazón de Jesús) nunca lograron ser espacios públicos ni perforar la manzana a totalidad.

El ideal de la ciudad moderna de Karl Brunner y de Manuel García recalcaba la importancia del concepto de pasaje como un espacio de transición en sus respectivos planes de desarrollo. Los pasajes acompañaron las propuestas de movilidad peatonal, además de convertirse en posibles espacios comerciales que conectarían la plaza fundacional con vías alternas. Sin embargo, ese ideal de pasaje perdió fuerza y para inicios del siglo xxi problemáticas como la densificación, la movilidad vehicular y el desempleo fueron temas más apremiantes que las propuestas del "Pasto Futuro". Entonces, los pasajes se quedaron guardados en los recuerdos de urbanistas y arquitectos que soñaron otro tipo de ciudad.

\section{REFERENCIAS}

Almandoz, A. (2007). Modernización urbanística en América Latina. Luminarias extranjeras y cambios disciplinares, 1900-1960. Iberoamericana, VII(27), 59-78. Recuperado dehttp://www.iai.spk-berlin.de/fileadmin/dokumentenbibliothek /lberoamericana/2007/27-Almandoz.pdf

Aguirre, B. (2009). Los pasajes y galerías comerciales de Santiago de Chile, como tránsito urbano hacia la modernidad. 1930-1960. Un proyecto patrimonial (tesis doctoral, Universidad de Sevilla, España). Recuperado de http:// dialnet.unirioja.es/servlet/tesis?codigo $=41748$

Álvarez, M. T. (2011). Karl Brunner en la ciudad de Pasto. En M. Álvarez, Manual de historia de Pasto, tomo XXII (pp. 126-157). Pasto: Academia Nariñense de Historia.

Álvarez, M. T. (2014). El cuarto centenario de la ciudad: ique sea un motivo! En M. Álvarez, Manual historia de Pasto, tomo XV (pp. 181-189). Pasto: Academia Nariñense de Historia.

Angulo, E. V. (1968). De la historia de Pasto. Cultura Nariñense, (35), 70-79.

Brunner, K. (1976). Embellecimiento de Pasto. Revista Cultural Nariñense (s. n.), 375-385

Choay, F. (1970). El urbanismo, utopías y realidades. Barcelona: Lumen.

Castro-Gómez, S. (2009). Tejidos oníricos: movilidad, capitalismo y biopolítica en Bogotá (1910-1930). Bogotá: Pontificia Universidad Javeriana. 
Delgado, R. (1938). Cooperación de todos los habitantes en el aseo y reforma de todos los edificios. Revista llustración Nariñense. VI, 6.

Espinoza, R. (2009). La ciudad de "Rutila". Pasto: Editorial Testimonio

Ficha predial Banco de Bogotá n. 001010200800005902 (s. f.). Bogotá: Instituto Geográfico "Agustín Codazzi", Catastro Nacional, Seccional Pasto.

Giedion, S. (1982). Espacio, tiempo y arquitectura. Madrid: Dossat S. A.

Gobernación de Nariño. (1997). Nariño, 93 años. Bogotá: Kimpres Ltda.

Hall, P. (1996). Ciudades del mañana. Historia del urbanismo en el siglo xx. Barcelona: Del Serbal.

Hoffer, A. (2003). Karl Brunner y el urbanismo europeo en América Latina. Bogotá: El Áncora editores.

Martínez, R. (2003). Manuel García 1928-1999. Arquitecto urbanista - semblanza. Recuperado el 12 de abril del 2013, de Red de revistas científicas de América Latina y el caribe, España y Portugal: http://www.redalyc.org/ articulo.oa?id=74810710

Real Academia Española: Diccionario de la lengua española, 23. ${ }^{a}$ ed., [versión 23.3 en línea]. Recuperado el 17 de mayo del 2020 de https://dle.rae.es

Una ciudad soñada. (14 de junio 1941). El Radio, p. 49.

Urresty, J. (2000). Historia urbana de Pasto. Bogotá: Testimonio. 
